\title{
Design and Development of a Novel Independent Wheel Torque Control of 4WD Electric Vehicle
}

\author{
Yue SHEN*, Bingnan ZHANG*, Hui LIU*, Yemin CUI**, Fida HUSSAIN*, Siwei HE*, \\ Fangyuan HU* \\ *School of Electrical and Information Engineering, Jiangsu University, XueFu Road, Jingkou District, Zhenjiang, China, \\ E-mail: amity@ujs.edu.cn, shen@ujs.edu.cn and fida.hussain07@yahoo.com \\ **R\&D Nantong Guangyi Mechanical \& Electrical Co. Ltd., Guangyi Road, Haian, Nantong, China, \\ E-mail: 287023542@qq.com \\ crossref http://dx.doi.org/10.5755/j01.mech.25.3.21879
}

\section{Introduction}

Development of electric vehicle is increasingly modern research in both academic and industrial fields [1]. All-wheel-drive systems [2] have been recognized as a break-through concept that will have a significant influence on the design of the future electric and hybrid vehicle [3]. Due to the use of hub motors [4], they have some advantages, such as packing flexibility, all-wheel-independent drive, space-saving, fast drivetrain response, etc. However, such electric vehicle needs accurate control of the driving forces on the steering wheels can function as power steering instead of the traditional engine-driven or electrical direct driven power steering system [5].

Several prior types of research have investigated the technology of steering or assisting steering by driving forces [6]. Francis Hooter and Meldrum firstly named skidsteering of wheeled combat vehicles as differential torque steer [7], but the critical point is that the combat vehicle does not have a steerable wheel for space-saving [8], so it is still skid-steering. Li et al. proposed a measure of assist steering [6] based on integrated steering and traction/braking system. The laboratory, led by professor Hori of the University of Tokyo, had developed two hub motor electric vehicle named "UOT Electric March I" and "UOT Electric March II" [911] . Then, they adopted the traditional steering and suspension system to realize the four-wheel independent drive/brake control by using the characteristics of hub motor that torque responses quickly and accurately, can be achieved easily and can be used individually to control each wheel $[10,11]$. The Swedish Volvo company proposed the concept of wheel independent component module (ACM) driven by hub motor and applied for a patent [12]. It focuses on how to integrate the driving, steering, braking, and suspension in each wheel part to a high degree of integration and the independent control of four-wheel integrated components by a computer to ensure riding comfort and handling stability, and to ensure that the tire is rolling resistance and wear minimal. The Chinese University of Hong Kong has developed a four-wheel drive/four-wheel steering multidirection moving a vehicle, which controls the drive and steering of the four wheels to achieve the in-place steering and lateral movement [13]. Nowadays, the four-wheel independent drive electric vehicles used in the field of agricultural [14] adopts differential steering mode, which has excellent wear on tires; or install a four-wheel stand-alone steering device, which requires greater control requirements and steering force. Neither of these two kinds of the electric vehicle can be very well driven in the farmland, especially in the thick mud.

In this paper, aimed to design a new hybrid vehicle chassis [15] without assisted steering system named as selfsteering four-wheel independent electric drive vehicle, the technical characteristics outlined is that there are two selfsteering mechanisms under the vehicle platform, and they can rotate around the fixed point in the platform. In this design, the following issues are addressed:

1) The problem of insufficient torque or insufficient mechanical strength [16], during steering is solved.

2) Abandoning the steering technology system. In this paper, the kinematics model and dynamic model of the newly designed vehicle are established by Newtonian mechanics. Then, introduce an improved self-steering fourwheel independent electric drive vehicle especially adding a linkage making the forward angle and the back angle equal.

Organization of the paper: In the next section, the kinematics model and dynamic model of the newly designed vehicle are established by Newtonian mechanics. Section 3 introduces an improved self-steering four-wheel independent electric drive vehicle especially adding a linkage making the forward angle and the back angle equal. Section 4 is devoted to the simulation experiment of the newly designed vehicle, and Section 5 concludes the paper.

\section{The structure designed and model established}

The vehicle chassis is one of the core components of the vehicle. Its capability directly determines the overall performance of the vehicle and plays a significant role in the design of the car. The operating conditions of farmland are different from those on the road, with high soil adhesion, more mud feet, difficult working conditions and high-performance requirements for working and moving mechanism.

\subsection{Structure and main parts of self-steering vehicle}

The vehicle platform consists of the following parts: 1 . the device of electric transmission 2. and 3. selfsteering mechanism, and the overall system is shown in Fig. 1.

In Fig. 2, the entire system consists of the following parts; gasoline engine, generator, rectifier inverter, battery storage system, and vehicle control unit. The hybrid system can provide better endurance comparatively exist- 
ence, recover excess energy and reduce the burden of braking system [17-19]. A gasoline engine was used to generate electricity. The generated 3-phase AC power converted into DC power by rectification. The DC power was used to drive hub-motor and battery storage.

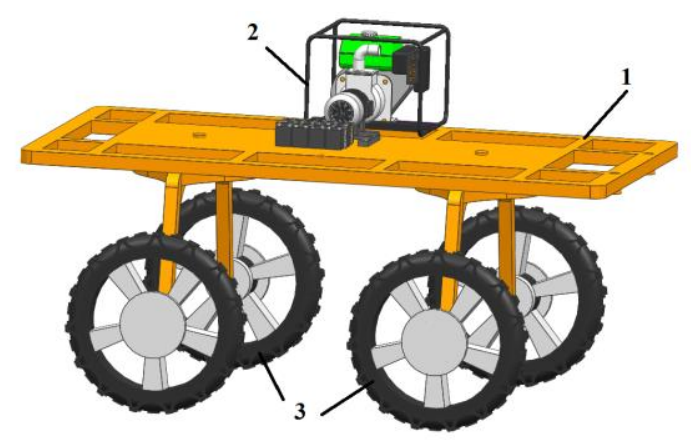

Fig. 1 Schematic of new self-steering vehicle chassis: 1 - vehicle platform, 2 - motors, electric power transmission, 3 - self-steering mechanism

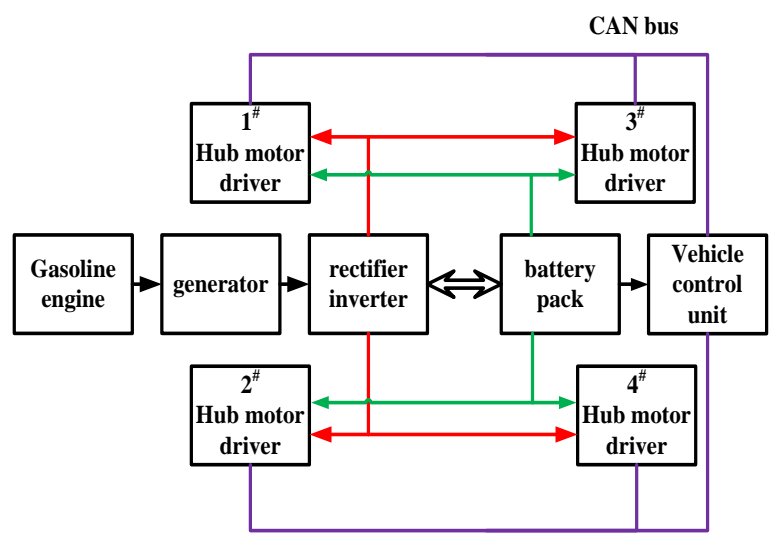

Fig. 2 Hybrid power sketches of the proposed vehicle system

Self-steering mechanism consists of bearing devices, support, and all four wheels a hub motor, and the two wheels' system is shown in Fig. 3. The top cylinder is fixed under the vehicle platform and can rotate around the center of the circle. The vehicle can steer only by controlling the speed of the wheels instead of driving bearing rotation, which is the origin of self-steering.

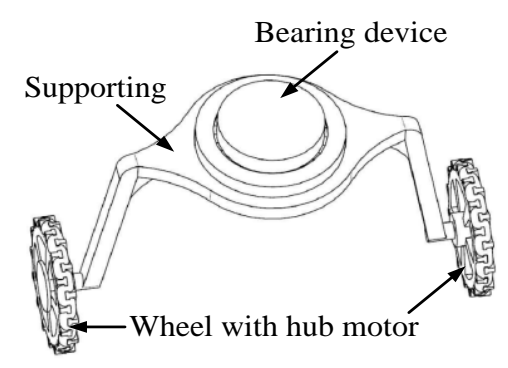

Fig. 3 Schematic diagram of the self-steering mechanism

\subsection{Model simplification of self-steering chassis}

The simplified model of the new self-steering chassis is shown in Fig. 3. The four rectangles represent the control system of four wheels. The horizontal segment represents the supporting and its fixed bearing, and the vertical portion represents the vehicle platform. The two parts of the front and rear parts can rotate around the two intersections.

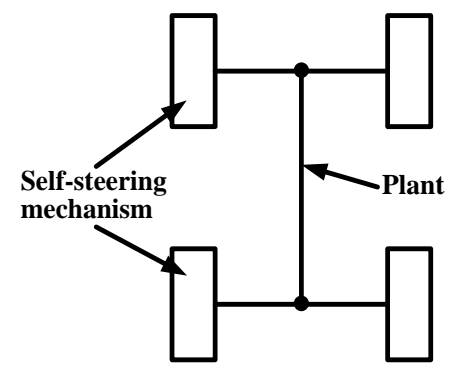

Fig. 4 Simplified model of the self-steering chassis

In the steering control system, the speed of the motors has a significate role. The direction of the vehicle can be directed by changing the speed of the four wheels. In the control of four hub-motors, first, a theoretical speed is needed. The rate of the hub-motors can be controlled by adjusting the duty cycle of the PWM pulse according to the theoretical speed and the real-time speed by feedback of the hub-motors.

\subsubsection{Kinematic analysis}

Kinematic analysis based on Ackermann-Jeantand steering model $[20,21]$. The Ackermann-Jeantand steering model is the most commonly used kinematics analysis method at present. Its characteristic is as follows: when the vehicle is in the steering process, the four wheels are circling a transient center with the same angular velocity. The steering model of only front wheel steering is shown in Fig. 4 when this characteristic is applied in the proposed vehicle's steering model.

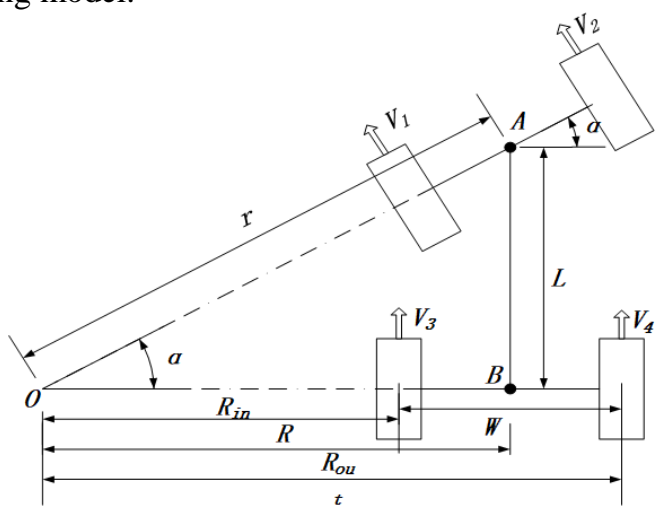

Fig. 5 Model with only front wheel steering

In Fig. 4, $\alpha$ is the turning angle of the vehicle, rad; $W$ is the distance between the left and right wheels of the vehicle, $\mathrm{m} ; L$ is the distance between the front and back wheels of the vehicle, $\mathrm{m} ; r$ is the turning radius of the front wheel of the vehicle, $\mathrm{m} ; R$ is the turning radius of the rear wheel of the vehicle, $\mathrm{m} ; R_{i n}$ is the turning radius of the rear inner wheel of the vehicle, $\mathrm{m} ; R_{\text {out }}$ is the steering radius of the rear lateral wheel of the vehicle, $m$.

In Fig. 4 , consider right angle triangle $\triangle \mathrm{OBA}$, the distance $L$ between the front and rear wheel may be calculated as:

$$
\tan \alpha=\frac{L}{R}
$$




$$
\sin \alpha=\frac{L}{r} .
$$

Assuming the overall speed of the vehicle is $V$ when it is in the steering process. The velocities of the four wheels may be calculated as follows:

$$
\begin{aligned}
& V=\frac{2 \pi R}{\Delta T}, \\
& V_{1}=\frac{2 \pi r-\pi W}{\Delta T}, \\
& V_{2}=\frac{2 \pi r+\pi W}{\Delta T}, \\
& V_{3}=\frac{2 \pi R-\pi W}{\Delta T}, \\
& V_{4}=\frac{2 \pi R+\pi W}{\Delta T} .
\end{aligned}
$$

The velocities of four wheels of the vehicle $V_{1}, V_{2}$, $V_{3}, V_{4}$ can get by substituting Eqs. (1), (2) and (3) in Eq. (4), (5), (6) and (7):

$$
\begin{aligned}
& V_{1}=V\left(\frac{1}{\cos \alpha}-\frac{W \tan \alpha}{2 L}\right), \\
& V_{2}=V\left(\frac{1}{\cos \alpha}+\frac{W \tan \alpha}{2 L}\right), \\
& V_{3}=V\left(1-\frac{W \tan \alpha}{2 L}\right), \\
& V_{4}=V\left(1+\frac{W \tan \alpha}{2 L}\right) .
\end{aligned}
$$

To get a smaller turning radius and make the vehicle more flexible during the steering process, the model of all-wheel steering can be set up, as shown in Fig. 5. Moreover, the functional relationship between the speed of each wheel, the speed of the vehicle and the steering angle of the model is established.

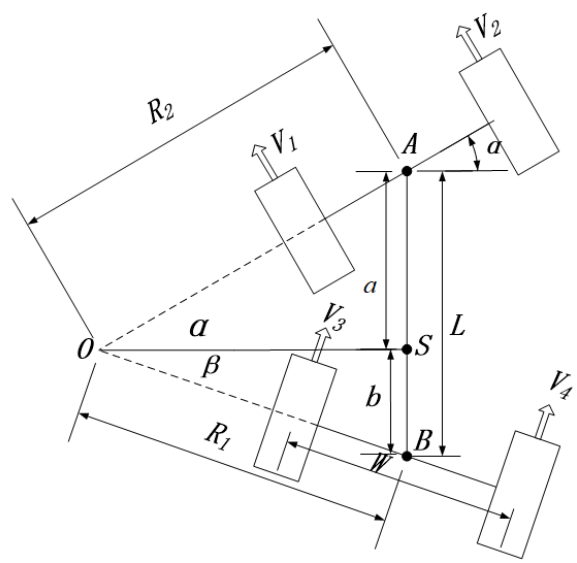

Fig. 6 Model with front and rear wheels steering
In the model in which the front and rear wheels are steering, the steering model is abstracted into two similar transient steering models (right angle triangle $\triangle \mathrm{OAS}$ and $\triangle \mathrm{OBS}$ ) by making a vertical line from the transient center to the body of the vehicle, $\mathrm{AB}$ is a vertical line and OS is a horizontal line of the vehicle. By deducing the characteristic function of the two transient models, the relation function of the speed of each wheel can be obtained based on Eqs. (8) (11):

$$
\begin{aligned}
& V_{1}=V\left(\frac{1}{\cos \alpha}-\frac{W \tan \alpha}{2 a}\right), \\
& V_{2}=V\left(\frac{1}{\cos \alpha}+\frac{W \tan \alpha}{2 a}\right), \\
& V_{3}=V\left(\frac{1}{\cos \beta}-\frac{W \tan \beta}{2 b}\right), \\
& V_{4}=V\left(\frac{1}{\cos \beta}+\frac{W \tan \beta}{2 b}\right) .
\end{aligned}
$$

Where, $\alpha$ i the steering angle of the ideal steering model in the front half of the vehicle, rad; $\beta$ - the steering angle of the ideal steering model in the rear half of the vehicle, rad.

In Fig. 5, consider right angle triangle $\triangle \mathrm{OAS}$ and $\triangle \mathrm{OBS}$, the length $a$ of the ideal steering model in the front half of the vehicle and the length $b$ of the ideal steering model in the front half of the vehicle may be calculated as:

$$
\begin{aligned}
& a=\frac{L \sin \alpha \cos \beta}{\sin (\alpha+\beta)}, \\
& b=\frac{L \sin \beta \cos \alpha}{\sin (\alpha+\beta)} .
\end{aligned}
$$

During steering control of the four wheels of the vehicle, it is necessary to balance the force of each wheel to make the vehicle move smoothly. However, the kinematics model has some limitations on steering analysis of the fourwheel drive vehicle [22]. Therefore, the dynamics model of the four-wheel vehicle steering is analyzed below.

\subsubsection{Dynamic analysis of the entire vehicle}

Newtonian mechanics are presented to analyze the dynamic system of the whole vehicle. In the process of steering control for the vehicle, the force condition of the entire vehicle is usually considered as a six degree of freedoms (SDOFs) force model [23] with translating in transverse, longitudinal, vertical and the revolving around the three perpendicular axes of each other. Certainly, the vehicle has three degrees of freedom assuming that the vehicle is moving on the platform. There are five freedoms in the model of this new vehicle chassis because two degrees of freedom of the two self-steering mechanisms are added. To facilitate the analysis, it is assumed that the factors such as 
vertical translation, pitching movement and lateral movement which have less influence on the steering movement of the vehicle, and can be ignored during the slower driving process. The force diagram is shown in Fig. 6.

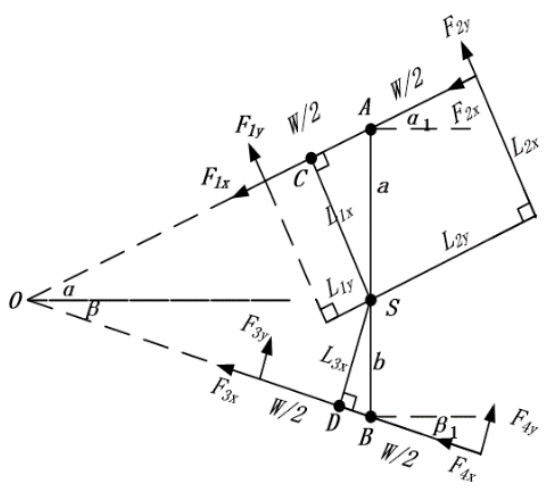

Fig. 7 Force analysis of 5-DOFs vehicle mode

In the force diagram $F_{1 \mathrm{x}}, F_{2 \mathrm{x}}, F_{3 \mathrm{x}}$ and $F_{4 \mathrm{x}}$ are the axial forces of four-wheels, $\mathrm{N} ; F_{1 \mathrm{y}}, F_{2 \mathrm{y}}, F_{3 \mathrm{y}}$ and $F_{4 \mathrm{y}}$ are the radial forces of the four-wheels, $\mathrm{N} ; m$ is the quality of the vehicle, $\mathrm{kg} ; V$ is the vehicle speed, $\mathrm{m} / \mathrm{s} ; \omega$ is the yaw rate and $I$ is the moment of inertia of the entire vehicle, $\mathrm{kg} \cdot \mathrm{m}^{2}$.

Assuming that the influence of air resistance and road slope on the vehicle is not considered, the kinematic equations of the five degrees of freedom can be expressed as follows:

Vertical equation:

$$
\begin{aligned}
& F_{1 y} \cos \alpha-F_{1 x} \sin \alpha+F_{2 y} \cos \alpha-F_{2 x} \sin \alpha+ \\
& +F_{3 y} \cos \beta+F_{3 x} \sin \beta+F_{4 y} \cos \beta+ \\
& +F_{4 x} \sin \beta=m \dot{V} \cos \theta-m \omega V \sin \theta .
\end{aligned}
$$

Horizontal equation:

$$
\begin{aligned}
& F_{1 y} \sin \alpha+F_{1 x} \cos \alpha+F_{2 y} \sin \alpha+F_{2 x} \cos \alpha- \\
& -F_{3 y} \sin \beta+F_{3 x} \cos \beta-F_{4 y} \sin \beta+ \\
& +F_{4 x} \cos \beta=m \dot{V} \sin \theta+m \omega V \cos \theta .
\end{aligned}
$$

Steering equation:

$$
\begin{aligned}
& I \dot{\omega}=F_{1 x} L_{1 x}-F_{1 y} L_{1 y}+F_{2 x} L_{2 x}+F_{2 y} L_{2 y}- \\
& -F_{3 x} L_{3 x}-F_{3 y} L_{3 y}-F_{4 x} L_{4 x}+F_{4 y} L_{4 y} .
\end{aligned}
$$

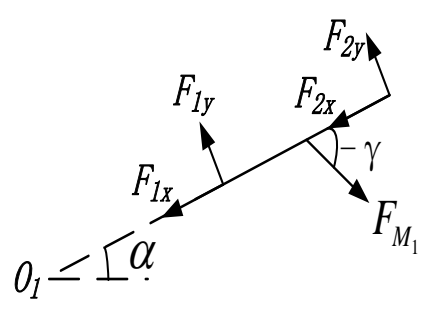

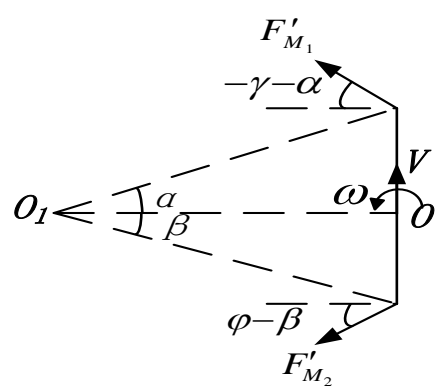

b
In Fig. 6, consider right angle triangles $\triangle \mathrm{SAC}$ and $\triangle \mathrm{SBD}$, the axial force torques $L_{1 \mathrm{x}}, L_{2 \mathrm{x}}, L_{3 \mathrm{x}}, L_{4 \mathrm{x}}$ and the radial force torques $L_{1 \mathrm{y}}, L_{2 \mathrm{y}}, L_{3 \mathrm{y}}, L_{4 \mathrm{y}}$ of four-wheels can be calculated as:

$$
\begin{aligned}
& L_{1 x}=L_{2 x}=a \cos \alpha, \\
& L_{3 x}=L_{4 x}=b \cos \beta, \\
& L_{1 y}=\frac{W}{2}-a \sin \alpha, \\
& L_{2 y}=\frac{W}{2}+a \sin \alpha, \\
& L_{3 y}=\frac{W}{2}-b \sin \beta, \\
& L_{4 y}=\frac{W}{2}+b \sin \beta .
\end{aligned}
$$

According to the above equations, it is easy to see that this is a system of five degrees of freedom. However, since equivalence relation is too weak, further Newton mechanics analysis for the model should be needed.

\subsubsection{Dynamic analysis of various parts of the vehicle}

Firstly, the model can be divided into three parts: the front self-steering mechanism, the middle body and rear self-steering mechanism. Then, the three components are analysis by Newtonian mechanics and rigid body rotation respectively. Finally, all equations are parameterized and sorted out.

The model of Fig. 6 can be divided into three parts and the following Fig. 7 is the force diagram of them.

Considered, the four parameters $F_{M_{1}}, F_{M_{2}}, F_{M_{1}}^{\prime}, F_{M 2}^{\prime}$ are added, these are the forces of front self-steering mechanism to the middle body and rear selfsteering mechanism to the middle body and their counterforce, N. And, $\gamma$ - the angle between $F_{M_{1}}$ and the front selfsteering mechanism, rad; $\varphi$ - the angle between $F_{M_{2}}$ and the rear self-steering mechanism, rad. (The counterclockwise is "+", clockwise as "-").

Fig. 8 Force analysis of three parts of the vehicle: $a$ - force analysis of front self-steering mechanism, $b$ - force analysis of the middle body, $\mathrm{c}$ - force analysis of rear self-steering mechanism 
In Fig. 7, according to the rotation of the rigid body and the second law of Newton, the following equation can be obtained.

Front self-steering mechanism:

$$
\begin{aligned}
& F_{1 y}\left(\frac{a}{\sin \alpha}-\frac{W}{2}\right)+F_{2 y}\left(\frac{a}{\sin \alpha}+\frac{W}{2}\right)+ \\
& +F_{M_{1}} \frac{a \sin \gamma}{\sin \alpha}=J_{1}^{\prime} \ddot{\alpha}, \\
& F_{1 x}+F_{2 x}-F_{M_{1}} \cos \gamma=m_{1} \dot{\alpha}^{2} \frac{a}{\sin \alpha} .
\end{aligned}
$$

Middle body:

$$
\begin{aligned}
& F_{M_{1}} \cos \gamma+F_{M_{2}} \cos \varphi=m_{2} \frac{V^{2} \tan \alpha}{a}, \\
& -F_{M_{1}} \sin (\gamma+\alpha)-F_{M_{2}} \sin (\varphi-\beta)=m_{2} \dot{V} .
\end{aligned}
$$

Rear self-steering mechanism:

$$
\begin{aligned}
& F_{3 y}\left(\frac{b}{\sin \beta}-\frac{W}{2}\right)+F_{4 y}\left(\frac{b}{\sin \beta}+\frac{W}{2}\right)+ \\
& +F_{M_{2}} \frac{b \sin \varphi}{\sin \beta}=J_{3}^{\prime} \ddot{\beta}, \\
& F_{3 x}+F_{4 x}-F_{M_{2}} \cos \varphi=m_{3} \dot{\beta}^{2} \frac{b}{\sin \beta} .
\end{aligned}
$$

In the above equations $J_{1}^{\prime}, J_{3}^{\prime}$ - the moment of inertia of the front self-steering mechanism and rear self-steering mechanism when they spin around the transient center of the vehicle, $\mathrm{kg} \cdot \mathrm{m}^{2}$. The moments can be formulated as:

$$
\begin{aligned}
& J_{1}^{\prime}=J_{1}+m_{1}\left(\frac{a}{\sin \alpha}\right)^{2}, \\
& J_{3}^{\prime}=J_{3}+m_{3}\left(\frac{b}{\sin \beta}\right)^{2} .
\end{aligned}
$$

Based on the law of parallel axis [24], $J_{1}, J_{3}$ - the moment of inertia of the front self-steering mechanism and rear self-steering mechanism when they spin around their geometric centers (barycenter), $\mathrm{kg} \cdot \mathrm{m}^{2}$.

The force of the entire chassis has fully analyzed, including friction, driving force and internal forces. Next, the above equations need to be sorted and simplified.

\section{The establishment and optimization of the model}

In order to validate the feasibility of the newly designed vehicle chassis system and its control effect, a 5SOFs electric vehicle dynamic model has been established. It can be easily seen that the new vehicle chassis is a multiinput and multi-output system [25].
For motor speed control, pulse width modulation (PWM) has many advantages, such as active noise immunity, saving space and economy [26]. In this paper, the PMW technique is used to drive the vehicle. The following formulae can be used to regulate the change of velocities $V_{i}(i=1,2,3,4$. $)$ so as to meet the requirements of forces $F_{i y}(i=1,2,3,4$. $)$ based on the model of a wheel in Fig. 8 [27], driving torque $T_{d}$ of each wheel and braking torque $T_{b}$ of each wheel can be calculated as:

$$
T_{d}-T_{b}=I_{\omega} \frac{\dot{V}_{i}}{R_{L}}-F_{i y} R_{L}, i=1,2,3,4
$$

Where, $I_{\omega}$ is the moment of inertia of each wheel and $R_{L}$ is wheel radius.

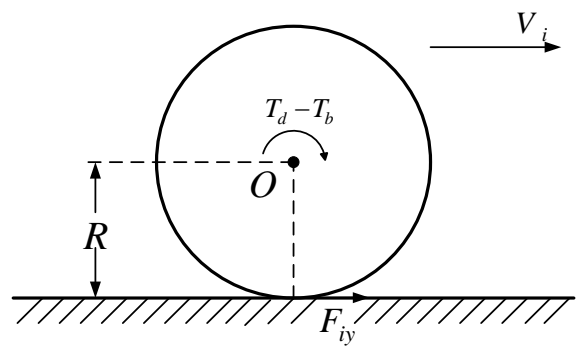

Fig. 9 The model of wheel

It can be seen from Eqs. (27-34) that the new vehicle chassis model is a strongly coupled nonlinear and incredibly complex system. In [28], using a unique linkage which making the rotation angle of the front self-steering mechanism and the rear self-steering mechanism the same such as a gearbox consist of four meshing gears. Then, the newly designed vehicle chassis model can be simplified as a 4DOFs model.

In Fig. 9, the gearbox fixed between the front steering mechanism and the rear steering mechanism. The two outer gears are fastened with two self-steering devices concentrically, and four-gears are set in a row and intermeshed with contiguous gear. It can be seen that this design makes the angle $\alpha$ of the front self-steering mechanism clockwise rotation and the angle $\beta$ of the rear self-steering mechanism counterclockwise rotations are equal.

There will be a torque almost nonexistent in the gearbox assuming the same angle of front and rear selfsteering mechanism by controlling faultlessly so that the new vehicle chassis model can be considered to be a 4DOFs model.

The equations (27)-(32) can be simplified as $\alpha=\beta$, $m_{1}=m_{3}$ and $J_{1}=J_{3}$ follows:

$$
\begin{aligned}
& \sin ^{2} \alpha\left(F_{1 y}+F_{2 y}-F_{3 y}-F_{4 y}\right)=m \omega V \sin \alpha- \\
& -m_{1} \dot{\alpha}^{2} L \cos \alpha-\frac{2 V^{2} \sin ^{2} \alpha}{L}, \\
& 2 I \dot{\omega} \tan \alpha+\dot{V}=\frac{\left(F_{1 y}+F_{2 y}+F_{3 y}+F_{4 y}\right) L}{\cos \alpha}- \\
& -W \tan \alpha\left(F_{1 y}-F_{2 y}+F_{3 y}-F_{4 y}\right),
\end{aligned}
$$




$$
\begin{aligned}
& m_{1} \dot{\alpha}^{2} L+m_{2} \dot{V}+\frac{2 V^{2} \tan \alpha}{L}+\frac{2 J_{1}^{\prime} \ddot{\alpha} \sin 2 \alpha}{L}= \\
& =-\frac{W \sin 2 \alpha}{2 L}\left(F_{1 y}-F_{2 y}+F_{3 y}-F_{4 y}\right)+ \\
& +\left(F_{1 y}+F_{2 y}+F_{3 y}+F_{4 y}\right) \cos \alpha,
\end{aligned}
$$

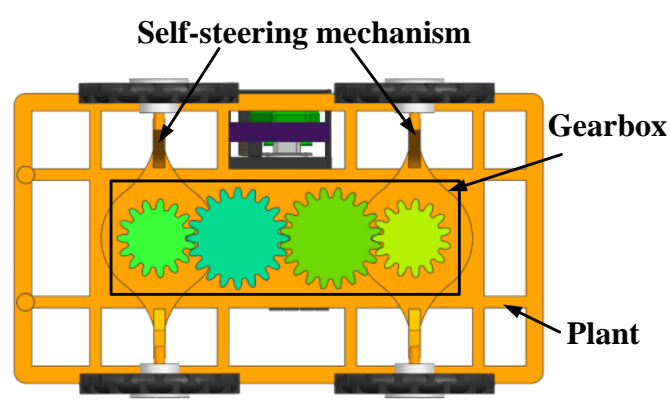

a

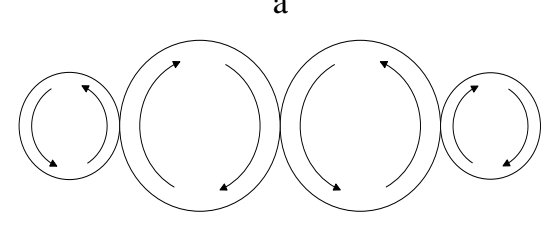

b

Fig. 10 Optimized simplification using linkage: a - optimized design sketch using linkage, $\mathrm{b}$ - an optimized schematic diagram using linkage

The above equations are the final model of the entire vehicle based on all kinds of analysis. Indeed, the feasibility and reliability of the vehicle must be verified first before the model is simulated.

\section{Simulation and experimental verification}

\subsection{Reliability verification by UG software}

UG software is a powerful CAD/CAE/CAM software, and its 3D structure modeling function is particularly prominent. The model of the new vehicle chassis was established using UG software. The system has been tested the effect of the vehicle in straight and turning. The reducing design cost, the reliability, and feasibility of the new vehicle chassis can be evaluated.

The performance of the model can be observed in two states: straight line and turn. First, the relationship between the rates of the four wheels can be determined in a satisfactory situation. The model schematic diagram of turning is shown in Fig.10, assumes that the speeds of the same side wheel are the same and the vehicle always turns around the same center.

The following equivalent relationship of $V_{1}, V_{2}, V_{3}$ and $V_{4}$ and can be found easily as:

$$
V_{1}\left(V_{3}\right): V_{2} V_{4}=l_{1}: l_{2}=(L-W \sin \alpha):(L+W \sin \alpha)
$$

The equations (12) -(15) are developed for the conclusion of kinematic analysis based on Ackermann-Jeantand steering model. Mark is created in the wheels to get the tracks of the four wheels on the vehicle when it is moving. The indentation of the front and rear wheels is represented by "." and " $\times$ " respectively so that the difference between the front and back wheels can be seen. The proposed models have been tested by simulation and developed the porotype and real model. The UG software simulation results are shown in Fig. 11, and the prototype, and real models are shown in Figs. 12 and 13 respectively.

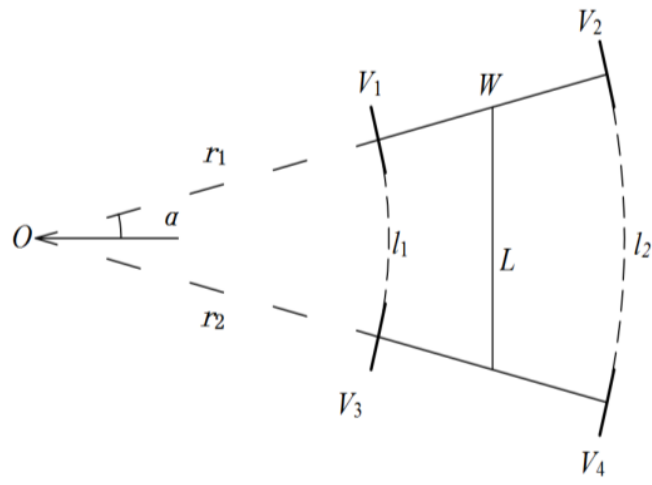

Fig. 11 Model schematic diagram of turning

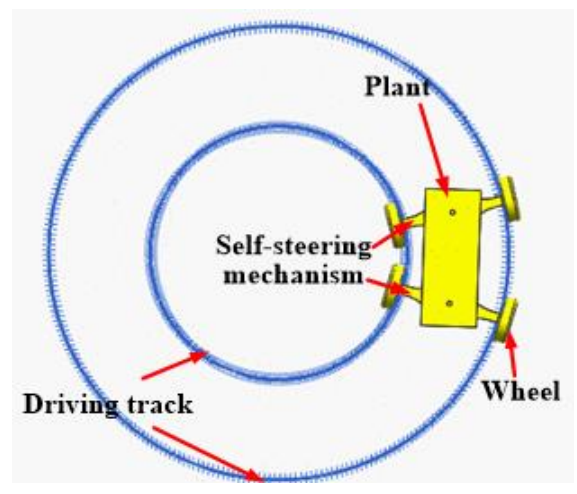

Fig. 12 The trajectories of four wheels of the proposed vehicle

The length, width, and height of the porotype of the proposed model (Fig.12) are $30 \mathrm{~cm}, 15 \mathrm{~cm}, 11.5 \mathrm{~cm}$ respectively. The length, width, and height of the real model (Fig.14) are $2 \mathrm{~m}, 1.3 \mathrm{~m}, 1.5 \mathrm{~m}$ respectively. It can be seen that the indentation of the wheels on both sides is the same track, and this is an advantage for the road and even the crops when the vehicle is driving. This characteristic can significantly reduce the damage to the driving environment. The simulation and experimental test verify that the reliability of the newly designed vehicle.

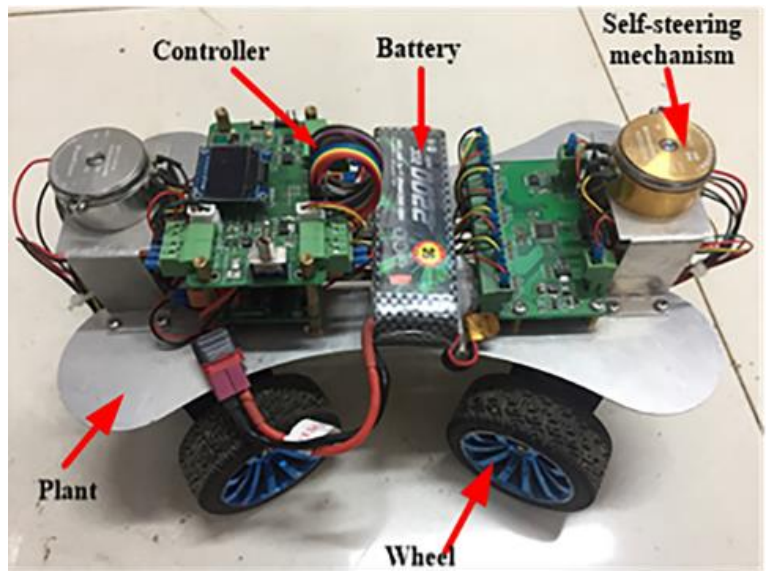

Fig. 13 The porotype of the proposed model 


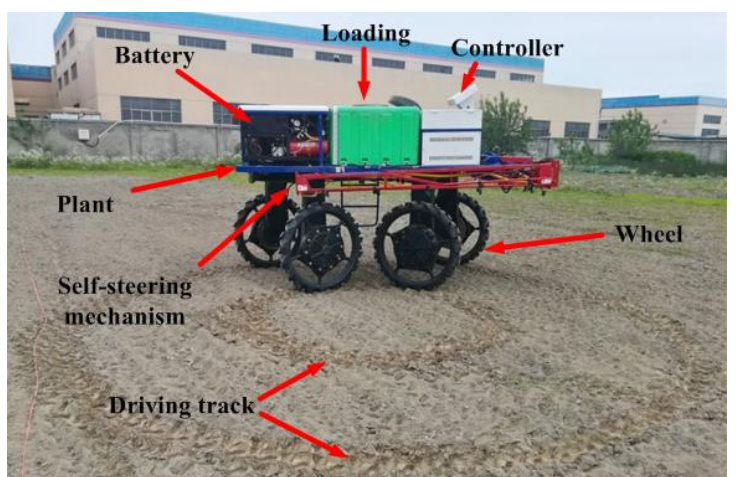

Fig. 14 The motion trajectory of the proposed real model

\subsection{MATLAB-based test}

In order to verify the superiority and controllability of the model, the simulation experiment of the model is carried out by MATLAB.

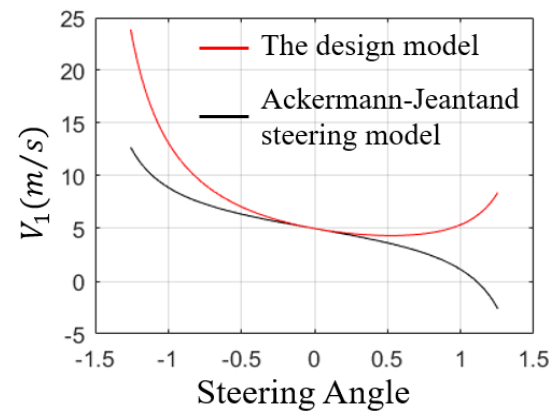

a

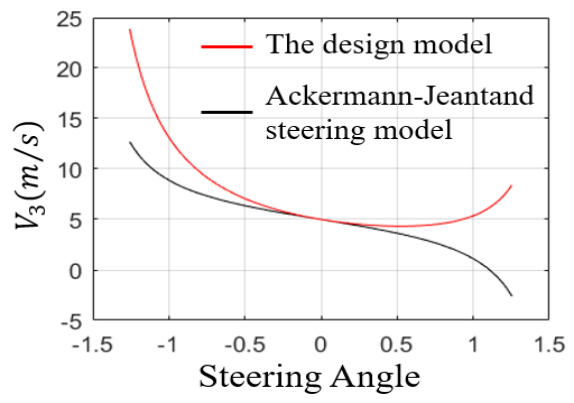

c
4.2.1. Superiority validation compared with AckermannJeantand steering model

According to the analysis of the previous Ackermann-Jeantand steering model and the design model, the simulation has been carried out using MATLAB.

The simulation parameters are used as follow: the distance between the front and back wheels of the vehicle $L=2 \mathrm{~m}$; the distance between the left and right wheels of the vehicle $W=1 \mathrm{~m}$.

In the simulation process, the steering angle is chosen from $-72^{\circ}$ to 72 (Assuming that the direction of the vehicle is $0^{\circ}$, the turning angle is positive when turning clockwise and negative when turning counter-clockwise). By choosing a given speed $V=5 \mathrm{~m} / \mathrm{s}$. The proposed model is compared with Ackermann-Jeantand steering model.

Theoretical speeds of four wheels under the Ackermann-Jeantand steering model and the design model are shown in Fig. 15.

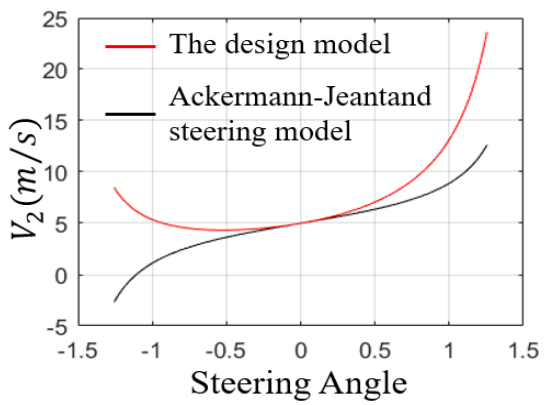

$\mathrm{b}$

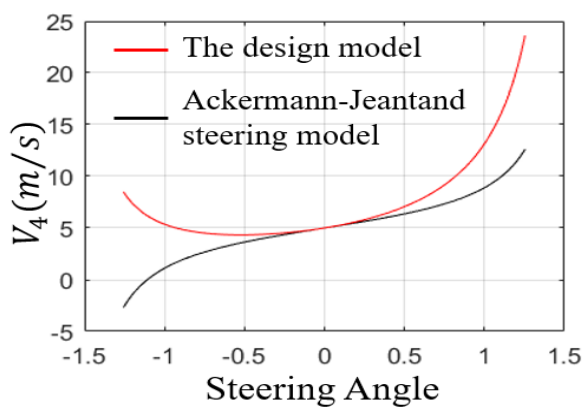

d

Fig. 15 Theoretical speed of four wheels: $\mathrm{a}$ - speeds of the front left wheel in two models, $\mathrm{b}$ - speeds of the front right wheel in two models, $\mathrm{c}$ - speeds of the rear left wheel in two models, $\mathrm{d}$ - speeds of the rear right wheel in two models

Figs. 15, a-d are the theoretical speed of four wheels (The identification of $V_{1}, V_{2}, V_{3}, V_{4}$ are shown in the Fig. 6), in which the red line represents the theoretical speed of the design model, and the black line represents the theoretical speed of the Ackermann-Jeantand, steering model. Under the circumstances of the same given speed, the conclusion can be obtained: comparing with the AckermannJeantand steering model, the range of adjusting speed is wider, so the control effect will be better. The greater advantage is that the vehicle designed have better performance: Ackermann-Jeantand steering model will have the negative speed when the vehicle is turning a bigger corner, which leads to more difficult and unstable the vehicle to be controlled. However, the vehicle designed in this paper always has a positive speed when turning large span, this characteristic makes this 4WD electric vehicle system more controllable.

\subsubsection{Open-loop control analysis of model}

The model was rebuilt based on Eqs. (28)-(30) to make a study of control on vehicle chassis and the output variables are determined to be $x, y, V$ and $\vartheta$, where, $x$, $y$ - the point coordinates of the center of the vehicle chassis in the geodetic coordinate system $O_{0} x_{0} y_{0}, V$ - the speed of the vehicle chassis, $\mathrm{m} / \mathrm{s}$; and $\vartheta$ - the angle of the $x_{0}$ axis of the geodetic coordinate system, rad. As shown in Fig. 16.

In Fig. 16, five state equations are listed as follows:

$$
\begin{aligned}
& \dot{x}=V \cos \vartheta, \\
& \dot{y}=V \sin \vartheta,
\end{aligned}
$$




$$
\begin{aligned}
& \ddot{\alpha}=\frac{\left(F_{1}-F_{2}\right) W}{2 J_{1}}=\frac{\left(F_{3}-F_{4}\right) W}{2 J_{1}}, \\
& m \omega V \sin \alpha-m_{1} \dot{\alpha}^{2} L \cos \alpha-\frac{2 V^{2} \sin ^{2} \alpha}{L}= \\
& =\sin ^{2} \alpha\left(F_{1 y}+F_{2 y}-F_{3 y}-F_{4 y}\right), \\
& \ddot{\vartheta}=\frac{L\left(F_{1 y}+F_{2 y}+F_{3 y}+F_{4 y}\right)}{2 I \sin \alpha}-\frac{L \dot{V}}{2 I \tan \alpha}- \\
& -\frac{W\left(F_{1 y}-F_{2 y}+F_{3 y}-F_{4 y}\right)}{2 I} .
\end{aligned}
$$

The control model of the vehicle chassis was built in MATLAB based on Eqs. (40-44) to make a study of control on vehicle chassis.

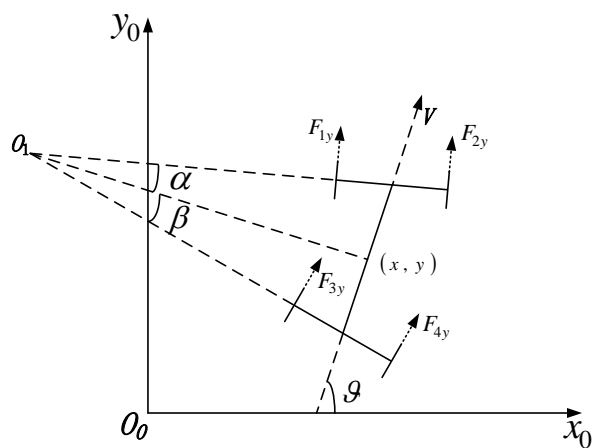

Fig. 16 Generalized coordinates of the vehicle chassis

Firstly, a special route was designed by MATLAB that includes five parts: three straight lines and two 1/4 circles connecting them and shown in Fig. 17.

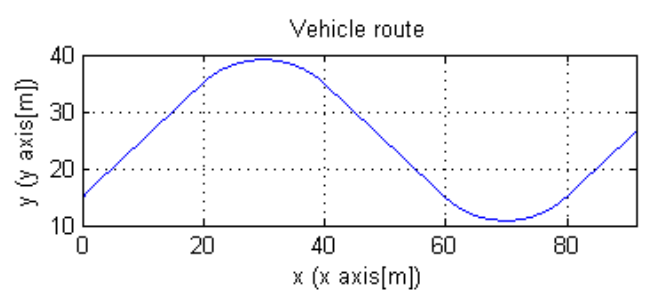

Fig. 17 The vehicle route of simulation by MATLAB

The waves forms of $F_{i y}(i=1,2,3,4$. $)$ about the time can be achieved assuming that the vehicle is tracking the route at a constant speed is shown in Fig. 18.
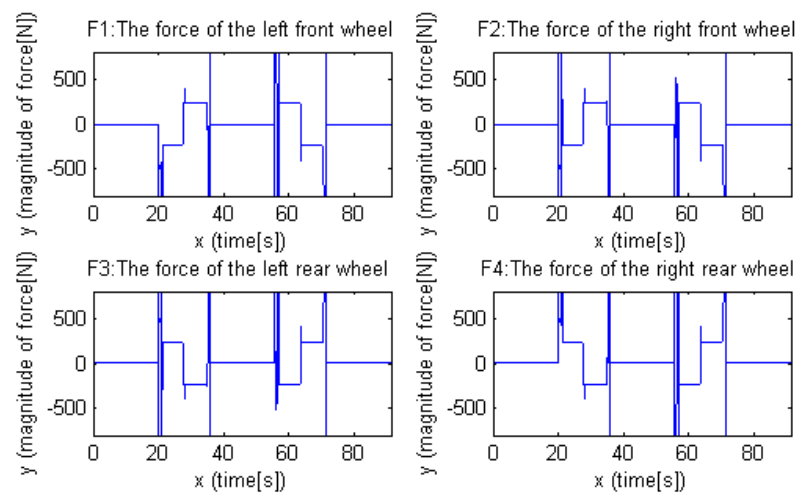

Fig. 18 The waveforms of the force of four wheels
The closed loop control system is used instead of open-loop control to achieve a better control effect on the vehicle. The PID control [30] algorithm was used to track the reference torque of 4 independent wheels in the vehicle. The vehicle enables to follow the trajectory accurately.

\section{Conclusion}

In this research work, a novel hybrid vehicle chassis without assisted steering and its model of the control system for the four-wheel-independent-drive electric vehicle was introduced. Both hardware and model parts of the innovative hybrid vehicle chassis system were designed and successfully implemented. The proposed model has been tested in the actual muddy agricultural field. The proposed design has the characteristics of simple structure, high transmission efficiency, energy saving, small turning force and other advantages such as it can turn around only keeping the different speed of the left and right wheels. Moreover, the control model of the new hybrid vehicle chassis was obtained from the kinematic model and dynamic models deducted by force analysis. The proposed model has been compared with Ackermann-Jeantand steering model and the proposed system show significantly higher results. Based on the independent drive of the electric vehicle, this different hybrid vehicle chassis has a broader speed range and an actual speed when turning great corners. These characteristics make the 4WD electric vehicle system more controllable. In addition, the damages of crops can be reduced using the proposed model because it has the same trajectory of both front and rear wheels. The proposed vehicle approach can be employed to a commercial and private four-wheel-independent-drive electric vehicle in the future.

\section{Acknowledgment}

This work was supported by Jiangsu International Science and Technology Cooperation Project [BZ2017067], Jiangsu Provincial Key Research and Development Program [BE2018372], Jiangsu Natural Science Foundation [BK20181443], Zhenjiang City Key Research and Development Program [NY2018001], Qing Lan project of Jiangsu Province and the Priority Academic Program Development (PAPD) of the Jiangsu Higher Education Institutions, China.

\section{References}

1. Liu Y.; Han Y.; Zhang B Z. 2012. Development of electric automobiles and electric vehicle charging facilities in China, Advanced Materials Research 424425:945-948.

2. Sakai, S. I.; Sado, H.; Hori, Y. 1999. Motion control in an electric vehicle with four independently driven inwheel motors, IEEE Trans of Mechatronics 4(1): 9-16.

3. Rizzoni, G.; Guzzella, L.; Baumann, B. M. 1999. Unified modeling of hybrid electric vehicle drivetrains, Mechatronics IEEE/ASME Transactions on 4(3): 246257.

4. Lu, D. B.; Ouyang, M. G.; Gu, J.; Li, J. Q. 2012. Field oriented control of permanent magnet brushless hub motor in electric vehicle, Electric Machines \& Control 16(11): 76-83.

5. Wang, J.; Wang, Q.; Jin, L.; Song, C. 2011. Independent wheel torque control of 4WD electric vehicle for differential drive assisted steering, 
Mechatronics 21(1): 63-76.

6. Kang, J.; Kim, W.; Lee, J.; Yi, K. 2010. Design, implementation, and test of skid steering-based autonomous driving controller for a robotic vehicle with articulated suspension, Journal of Mechanical Science \& Technology 24(3): 793-800.

7. Hirano, Y. 2016. Model based development of an integrated control of front steering and torque vectoring differential gear system, 2016 55th Annual Conference of the Society of Instrument and Control Engineers of Japan (SICE): 11-16,

8. Bianchi, D. 2010. Adaptive integrated vehicle control using active front steering and rear torque vectoring, IEEE Conference on Decision \& Control: 3557-3562.

9. Hori, Y.; Toyoda, Y.; Tsuruoka, Y. 1998. Traction control of electric vehicle: basic experimental results using the test EV "UOT electric march, Industry Applications IEEE Transactions 34(5): 1131-1138.

10. Hori, Y. 2004. Future vehicle driven by electricity and control-research on four wheel motored UOT Electric March II, IEEE Transactions 51(5): 954-962.

11. Hori, Y. 2002. Future vehicle driven by electricity and control-research on four wheel motored "UOT Electric March II", in International Workshop on Advanced Motion Control: 1-14.

12. Lu, D.; Li, J.; Ouyang, M.; Gu, J. 2011. Research on hub motor control of four-wheel drive electric vehicle, in Vehicle Power and Propulsion Conference: 1-5.

13. Yu, S. H.; Moskwa, J. J. 1994. A global approach to vehicle control: coordination of four wheel steering and wheel torques, Journal of Dynamic Systems Measurement \& Control 116(4): 659-667.

14. Song, S.; Qu, J.; Li, Y.; Guo, K.; Wei, Z. 2015. Experiment on movement and dynamic property of driving wheel with offset steering shaft for agricultural vehicle, Transactions of the Chinese Society of Agricultural Engineering 31(23): 28-34.

15. Kim, D.; Hwang, S.; Kim, H. 2008. Vehicle stability enhancement of four-wheel-drive hybrid electric vehicle using rear motor control, IEEE Transactions on Vehicular Technology 57(2): 727-735.

16. Zhang, K.; Yu, H.; Ding, X. 2018. Experimental validation of active holographic metasurface for electrically beam steering. Optics Express 265(5): 6316.

17. Antsaklis, P. J.; Stiver, J. A.; Lemmon, M. D. 1993. Hybrid System Modeling and Autonomous Control Systems, in Hybrid Systems: 366-392.

18. Yamaguchi, K.; Hisada, H. 1998. Hybrid vehicle: 4750.

19. Sciarretta, A.; Guzzella, L. 2007. Control of hybrid electric vehicles, Control Systems IEEE 27(2): 60-70.

20. Ackermann, J.; Guldner, J.; Sienel, W.; Steinhauser, R. 1995. Linear and nonlinear controller design for robust automatic steering, IEEE Transactions on Control Systems Technology 3(1): 132-143.

21. Bascetta, L.; Cucci, D. A.; Matteucci, M. 2016. Kinematic trajectory tracking controller for an all-terrain Ackermann steering vehicle, Ifac Papersonline 49(15): 13-18.

22. Chatzikomis; C. I, Spentzas. 2014. Comparison of a vehicle equipped with Electronic Stability Control (ESC) to a vehicle with Four Wheel Steering (4WS)[J]. Forschung Im Ingenieurwesen 78(1-2): 13-25.

23. Loof, J.; Besselink, I..; Nijmeijer, H.. 2018.
Implementation and validation of a three degrees of freedom steering-system model in a full vehicle model, Vehicle System Dynamics: 1-22.

24. Sun, K. K.; Zhu, Z. B. 2017. Calculation of moment of inertia of Light metal eccentric crankshaft based on parallel axis theorem, World Nonferrous Metals 8(1): 60-61.

25. Zhang, Q. 2002. Adaptive observer for multiple-inputmultiple-output (MIMO) linear time-varying systems, Automatic Control IEEE Transactions on 4(3): 525-529.

26. Radhika, V.; Baskaran, K. 2017. Hybrid digital pulse width modulator architecture using FPGA, IEEE International Conference on Recent Trends in Electronics: 123-126.

27. Baghani, A.; Ahmadabadi, M. N.; Harati, A. 2006. Kinematics Modeling of a Wheel-Based Pole Climbing Robot (UT-PCR), in IEEE International Conference on Robotics and Automation: 2099-2104.

28. Tomizuka, M. 2002. Mechatronics: from the 20th to 21st century, Control Engineering Practice 10(8): 877886.

29. Ang, K. H.; Chong, G.; Li, Y. 2005. PID control system analysis, design, and technology, IEEE Transactions on Control Systems Technology 13(4): 559-576.

30. Astrom, K J.; Hagglund, T. 2001. The future of PID control, Control Engineering Practice 9(11): 1163-1175.

Y. Shen, B. Zhang, H. Liu, Y. Cui, F. Hussain, S. He, F. Hu

DESIGN AND DEVELOPMENT OF A NOVEL INDEPENDENT WHEEL TORQUE CONTROL OF 4WD ELECTRIC VEHICLE

S u m m a r y

In this paper, a novel hybrid vehicle chassis is designed without an assisted steering system and its kinematics model, the dynamics model, and the control system. The existing steering mechanism does not provide enough torque or has a shortage of mechanical strength and control when sinking into deeper muddy road. The proposed design can solve these problems smoothly. Initially, the new 4wheel-drive (4WD) hybrid vehicle chassis vehicle platform and self-steering mechanism are designed, and Its kinematics and dynamics models are built. The linkages are added between the front and rear bodies to optimize the control model. Finally, traditional proportion-integration-differentiation (PID) feedback control loop is employed to track the reference torque of 4 independent wheels. The new hybrid vehicle chassis steering system has been tested in the actual muddy agricultural field. The results are verified using a prototype and real vehicle. The proposed design is proved feasibility not only has a smaller turning radius but also has a higher control precision than an existing vehicle, which ensures the flexibility of the vehicle and can control the direction as well. Besides, the proposed design is best for modern agricultural vehicle and can be implemented in commercial and private vehicles.

Keywords: 4WD electric vehicle, hybrid vehicle, steeringless vehicle.

Received October 26, 2019

Accepted June 14, 2019 